\title{
Inverse spin-Hall effect voltage generation by nonlinear spin-wave excitation
}

\author{
Laura Feiler, ${ }^{1, *}$ Kathrin Sentker, ${ }^{1}$ Manuel Brinker, ${ }^{1}$ Nils Kuhlmann, ${ }^{1}$ Falk-Ulrich Stein, ${ }^{2}$ and Guido Meier ${ }^{1,2,3}$ \\ ${ }^{1}$ Institut für Nanostruktur- und Festkörperphysik, Universität Hamburg, Jungiusstrasse 11, 20355 Hamburg, Germany \\ ${ }^{2}$ Max-Planck Institute for the Structure and Dynamics of Matter, Luruper Chaussee 149, 22761 Hamburg, Germany \\ ${ }^{3}$ Center for Free-Electron Laser Science (CFEL), Luruper Chaussee 149, 22761 Hamburg, Germany
}

(Received 1 July 2015; revised manuscript received 7 January 2016; published 4 February 2016)

\begin{abstract}
We investigate spin currents in microstructured permalloy/platinum bilayers that are excited via magnetic high-frequency fields. Due to this excitation spin pumping occurs at the permalloy/platinum interface and a spin current is injected into the platinum layer. The spin current is detected as a voltage via the inverse spin-Hall effect. We find two regimes reflected by a nonlinear, abrupt voltage surge, which is reproducibly observed at distinct excitation field strengths. Micromagnetic simulations suggest that the surge is caused by excitation of a spin-wave-like mode. The comparatively large voltages reveal a highly efficient spin-current generation method in a mesoscopic spintronic device.
\end{abstract}

DOI: 10.1103/PhysRevB.93.064408

\section{INTRODUCTION}

The demand for alternative technologies rises, due to the ongoing miniaturization of electronic devices. Promising ideas can be found in the field of spintronics. Here not only the charge current, but also the spin current is utilized, taking into account the spin as an additional degree of freedom. Since charge currents lead to problematic Joule heating in devices the idea to generate a pure spin current, using a spin battery, was proposed by Brataas et al. [1]. The precessing magnetization $\vec{m}$ of a ferromagnet at resonance (FMR) in contact with a normal metal generates a spin accumulation at the interface that diffuses into a normal metal [2-5], or as recently shown into a semiconductor [6]. This process is denoted as spin pumping. The resulting spin current $\vec{j}_{\mathrm{s}}$ in the normal metal can be described as $[1,4]$

$$
\vec{j}_{\mathrm{s}}=\frac{\hbar}{4 \pi} g^{\uparrow \downarrow}\left(\vec{m} \times \frac{d \vec{m}}{d t}\right),
$$

with the real part of the spin mixing conductance $g^{\uparrow \downarrow}$ that summarizes the spin-dependent transport properties of the interface [1]. There are various methods to detect this spin current, e.g., by measuring the enhanced damping $[2,3]$ or the backflow of the spin current [7] in the ferromagnet, utilizing the Rashba effect [8], or by measuring the inverse spin-Hall effect (ISHE) $[9,10]$. In the latter case the strong spin-orbit interaction scatters the two spin orientations of the spin current perpendicular to their direction of motion in heavy metals like platinum. For a nonvanishing spin polarization of the spin current $\vec{\sigma}$ this gives rise to a charge current $\vec{j}_{\mathrm{c}}[9,10]$, cf. Fig. 1(a):

$$
\vec{j}_{\mathrm{c}}=\Theta_{\text {ISHE }} \frac{2 e}{\hbar}\left(\vec{j}_{\mathrm{s}} \times \vec{\sigma}\right),
$$

whereby $\Theta_{\text {ISHE }}$ is the spin Hall angle.

To investigate the influence of the high-frequency excitation field on the created spin current, we study laterally microstructured permalloy $\left(\mathrm{Ni}_{80} \mathrm{Fe}_{20}\right)$ /platinum $(\mathrm{Pt})$ bilayers, see Fig. 1. Note, that the platinum element is slightly wider

*Ifeiler@physnet.uni-hamburg.de than the permalloy element in order to avoid a direct contact between ferromagnet and voltage contacts and, thus, parasitic effects. The magnetization of the permalloy layer is excited via FMR and a spin current is generated at the interface that is detected as a voltage via the ISHE. Similar transport measurements have been reported before, e.g., in Refs. [9-17]. In these publications a linear (quadratic) relation between the excitation power (field amplitude) and the measured voltage was found. The bilayers investigated in our experiment are one order of magnitude smaller in the lateral dimension and, thus, the conversion from spin current to charge current is being conducted within a length of only $1 \mu \mathrm{m}$. Also, the coplanar waveguides that are used for FMR excitation are one order of magnitude narrower (3.6 $\mu \mathrm{m}$ wide signal conductor). This enhances the amplitude of the high-frequency Oerstedt field and thereby the ISHE voltage generation. The higher excitation fields reveal a regime of increased spin-current generation, that is reflected by an abrupt, nonlinear voltage surge at a distinct excitation amplitude. Recent studies by Bauer et al. show that in permalloy structures nonlinear spin-wave excitation occurs at FMR above a critical high-frequency excitation field [18]. This effect can be linked with our measurements via micromagnetic simulations and our FMR measurements. In the strong excitation regime, the current density reached in our mesoscopic device is two orders of magnitude larger than the ones in the conventional millimeter-sized ISHE experiments [9-17].

\section{SAMPLE PREPARATION}

The samples are prepared via electron-beam lithography and lift-off processing. At first a 120-nm-thick coplanar gold waveguide (CPW) is thermally evaporated on top of a $\mathrm{SiO}_{2}$ substrate, as shown in Fig. 1(b). Then a 120-nm-thick layer of hydrogen silsesquioxane (HSQ) is deposited to prevent an electrical short between $\mathrm{CPW}$ and voltage contacts that are deposited later. In the next step, an ensemble of $8-\mu \mathrm{m}$ long, $2-\mu \mathrm{m}$-wide, and $30-\mu \mathrm{m}$-thick permalloy rectangles is thermally evaporated on top of the signal conductor of the CPW. This ensemble enhances the signal strength of the broadband-FMR signal. On top of each of these rectangles a 15-nm-thick platinum strip is deposited via dc-magnetron 

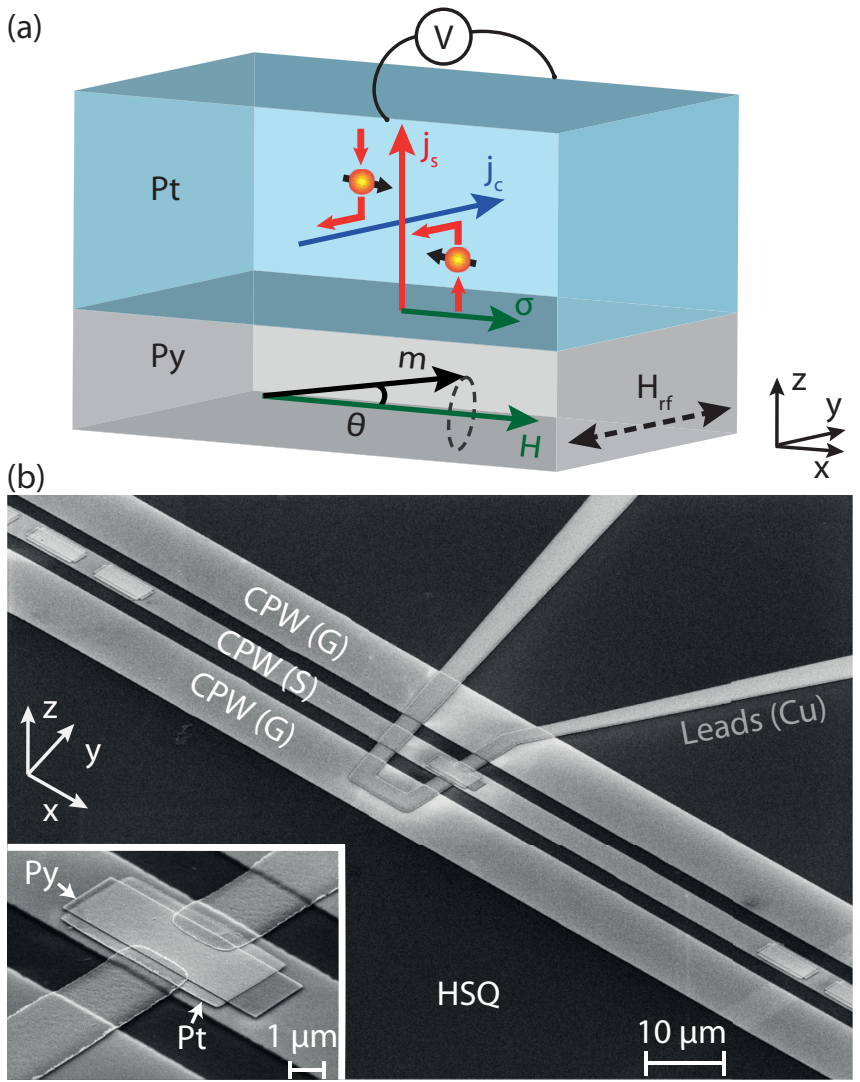

FIG. 1. (a) Sketch of the Py/Pt bilayer. The magnetization $\vec{m}$ precesses around the static magnetic field $\vec{H}$ with the cone angle $\theta$. The spin polarization $\vec{\sigma}$ is aligned parallel to $\vec{H}$. A spin current $\vec{j}_{s}$ is pumped into the platinum layer that is converted into a charge current $\vec{j}_{c}$ via the ISHE. (b) Scanning-electron micrograph of a Py/Pt bilayer sample. The inset shows a closeup of the center region. The bilayer is placed on top of the insulating HSQ over the signal conductor of the CPW. The copper leads are connecting the bilayer to a voltmeter.

sputtering. Then 100-nm-thick, sputtered copper leads are added as voltmeter contacts to one double layer in the center of the CPW, as depicted in the inset of Fig. 1(b). Prior to every sputter deposition step the sample is cleaned in situ via rf argon-plasma etching. To be able to determine the influence of spin-rectification effects on our measurements, we have prepared reference samples without the platinum layer. We will address the sample types as Py/Pt and Py/-, respectively. All measurements are performed at room temperature.

\section{MEASUREMENTS AND RESULTS}

In order to ensure that the measured voltage is generated by the spin-pumping effect, we characterize the magnetization dynamics of the permalloy rectangles via broadband-FMR spectroscopy with a vector-network analyzer. A static magnetic field $H$ is applied along the $x$ axis, cf. Fig. 1, which is varied from $\mu_{0} H=-90 \mathrm{mT}$ to $90 \mathrm{mT}$. For every field step an additional high-frequency magnetic field $H_{\mathrm{rf}}$, aligned along the $y$ axis, is applied via the waveguide, while the transmission through the signal line of the CPW is measured ten times and then averaged. After every field step a reference signal of $\mu_{0} H=90 \mathrm{mT}$ is applied along the $y$ axis, so that the static magnetic field $H$ and the high-frequency magnetic field $H_{\mathrm{rf}}$ are aligned parallel and, thus, no FMR signal can occur. This reference signal is also detected ten times for every frequency and then averaged. Taking the difference $\Delta T$ of the measurement signal and the reference signal for every field step drastically decreases parasitic contributions like reflections in the setup and the frequency dependence of the transmission. The transmission parameter $S_{12}$ is measured. At port i the VNA measures the incoming power $P_{i, \text { in }}$ and outgoing power $P_{i, \text { out }}$. Therefore, $S_{12}$ is defined as

$$
S_{12}=10 \log \left(\frac{P_{1, \text { in }}}{P_{2, \text { out }}}\right)_{P_{1, \text { out }=0}}, \quad\left[S_{12}\right]=\mathrm{dB} .
$$

With this $S$ parameter, an impedance of $R \sim 50 \Omega$, the width $w$ of the signal line and the rf current $I_{\mathrm{rf}}$ an estimation of the minimal applied high-frequency field strength is possible $[19,20]$ :

$$
\begin{aligned}
H_{\mathrm{rf}} & =\frac{I_{\mathrm{rf}}(t)}{2 w}=\frac{1}{2 w} \sqrt{\frac{2}{50 \Omega} P_{2, \mathrm{out}}} \\
& =\frac{1}{2 w} \sqrt{\frac{2}{50 \Omega} P_{1, \text { in }} 10^{S_{12} /(d B \cdot 10)}} .
\end{aligned}
$$

The rf field depends on the width $w$ of the signal line, on the incoming power $P_{1 \text {,in }}$ used for excitation, and on the damping of the system. Contributing to this overall damping are the measurement setup and the individual damping of each waveguide depending on the excitation frequency and excitation power of the high-frequency field. Thus, the highfrequency field has to be determined with the correct value of $S_{12}$ for every measurement. For the measurements performed using only one excitation frequency and excitation power of the high-frequency field, this value is easy to determine, while for the complete broadband FMR measurements, an averaged value is used.

For the measurement displayed in Fig. 2(a), the dominant absorption line is the FMR mode of the permalloy rectangles $[21,22]$. In the measurement black (white) indicates low (high) transmission and, thus, high (low) energy absorption of the magnetic microstructures. The modes at higher frequencies are spin-wave surface modes (SW-S) [22,23] and the ones at lower frequencies are spin-wave edge modes (SW-E) [24]. Figure 2(a) also shows a horizontal and a vertical profile through the transmission data. Like typical absorption phenomena, the transmission obeys a Lorentzian shape. Following Kalarickal et al. [25], such a broadband-FMR measurement can be used to determine the Gilbert damping constant $\alpha$. Therefore, we fit every vertical line profile with a Lorentzian and determine the frequency-dependent peak width at halfmaximum $\Delta f$ and the resonance frequency for every field step. The resonance frequencies are used to perform a fit with the Kittel formula [25,26], cf. green line in Fig. 2(a), extracting the saturation magnetization $M_{\mathrm{s}, \mathrm{Py} / \mathrm{Pt}}=(835 \pm 26) \mathrm{kA} \mathrm{m}^{-1}$ and the anisotropy field $H_{\mathrm{k}, \mathrm{Py} / \mathrm{Pt}}=(10 \pm 3) \mathrm{kA} \mathrm{m}^{-1}$. Figure 2(b) shows the frequency-dependent peak width at half-height $\Delta f$. The Gilbert damping constant $\alpha$ and the inhomogeneous broadening in a magnetic field due to sample 

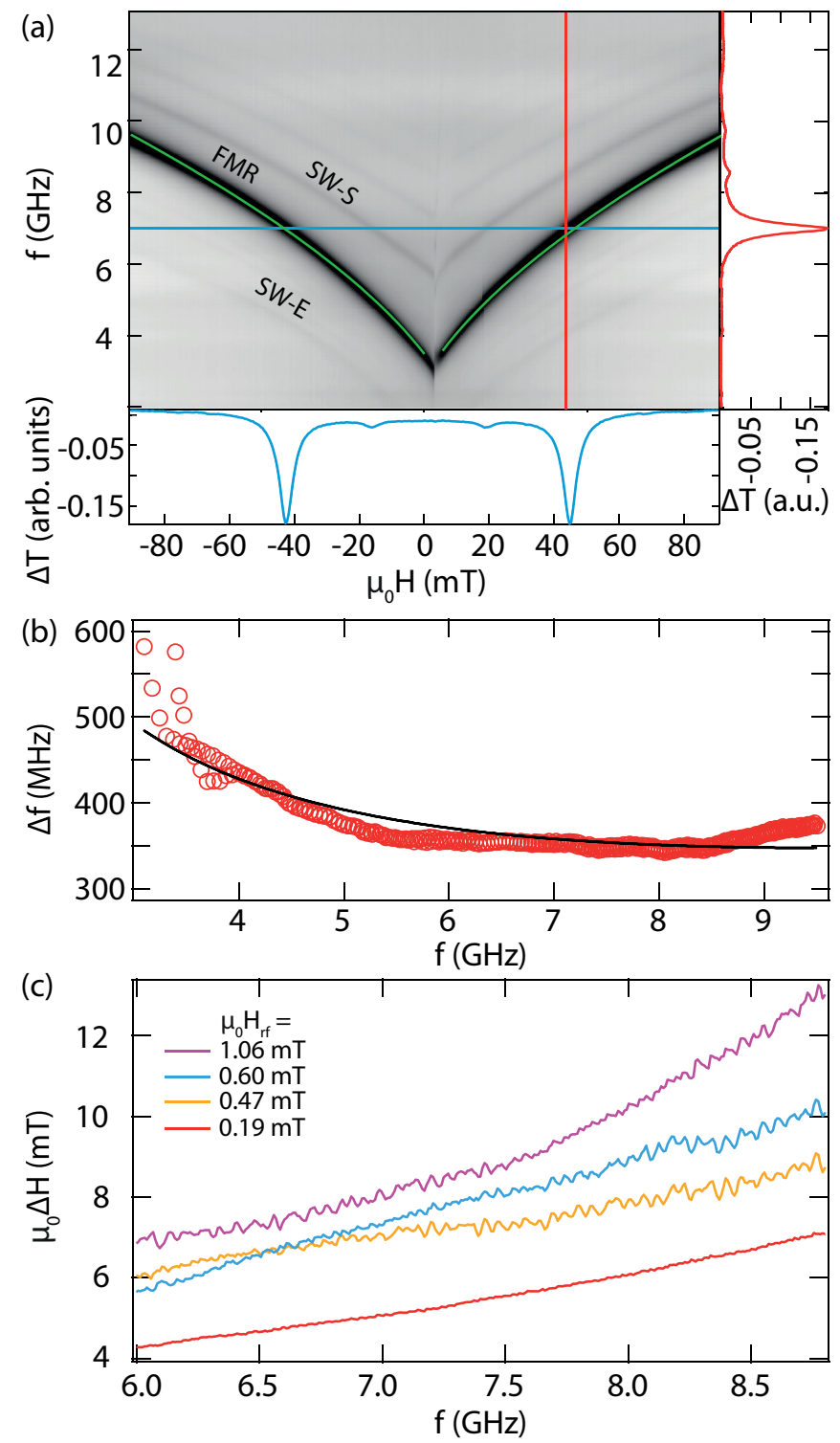

FIG. 2. (a) Broadband-FMR spectroscopy of a Py/Pt bilayer for $\mu_{0} H_{\mathrm{rf}}=0.19 \mathrm{mT}$. Low (high) transmission $T$ through the waveguide is indicated by black (white) color. A horizontal profile (blue) and a vertical profile (red) is shown. The green line is a Kittel fit. (b) Peak width at half-height $\Delta f$ as a function of the frequency, obtained by the frequency-dependent Lorentzian fits for every field step. The black line is a fit using Eq. (5). (c) Peak width at half-maximum $\Delta H(f)$ for different high-frequency fields $H_{\mathrm{rf}}$ indicated in the figure.

imperfections $\Delta H_{0}$ is calculated as follows [25,26]:

$$
\Delta f=\left(\frac{\gamma \mu_{0}}{2 \pi} \Delta H_{0}+2 \alpha f\right) \sqrt{1+\left(\frac{\gamma \mu_{0} M_{\mathrm{s}}}{4 \pi f}\right)^{2}},
$$

whereby $\gamma=176 \mathrm{GHz} \mathrm{T}^{-1}$ is the gyromagnetic ratio. For an excitation field of $\mu_{0} H_{\mathrm{rf}}=0.19 \mathrm{mT}$ the value for a permalloy/platinum bilayer sample is $\alpha_{\mathrm{Py} / \mathrm{Pt}}=0.0069 \pm 0.0001$, while for a sample without the platinum layer we determine $\alpha_{\text {Py/- }}=0.0046 \pm 0.0001$. The difference is caused by the additional energy loss in the Py/Pt bilayer sample due to the spin-pumping effect [9]. We evaluated the broadband

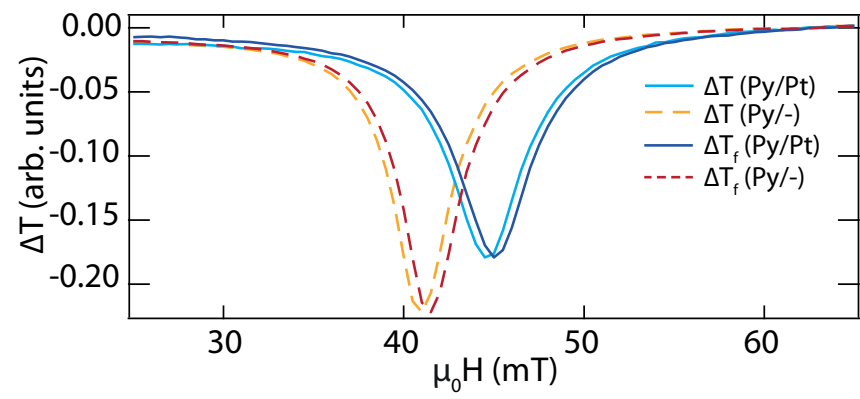

FIG. 3. Comparison of field reference and frequency reference method: Transmission spectra $\Delta T$ for a constant frequency of $7 \mathrm{GHz}$ and $\mu_{0} H_{\mathrm{rf}}=0.19 \mathrm{mT}$ for one sample with (cyan line) and one without (dashed yellow line) a Pt layer, using a reference field of $\mu_{0} H=90$ $\mathrm{mT}$ that is aligned along the $y$ direction. In addition the transmission spectra $\Delta T_{\mathrm{f}}$ for a constant frequency of $7 \mathrm{GHz}$ and $\mu_{0} H_{\mathrm{rf}}=0.19$ $\mathrm{mT}$ for one sample with (blue line) and one without (dashed red line) a Pt layer are displayed, obtained with a reference frequency of $f_{\text {ref }}=2 \mathrm{GHz}$. The transmission spectra $\Delta T_{\mathrm{f}}$ are shown without offsets.

FMR measurement with the high-frequency field strength $\mu_{0} H_{\mathrm{rf}}=0.19 \mathrm{mT}$ in order to connect it directly to the ISHE measurements shown in Fig. 5. In addition we evaluated the damping for a low excitation field of $\mu_{0} H_{\mathrm{rf}}=0.06 \mathrm{mT}$, because it yields the linear Gilbert damping constant [27], see also Fig. 2(c). Here the determined value for a sample with a permalloy/platinum bilayer is $\alpha_{\mathrm{Py} / \mathrm{Pt}}=0.0089 \pm 0.00004$, while for a sample with only a permalloy layer $\alpha_{\mathrm{Py} / \mathrm{-}}=$ $0.0071 \pm 0.00006$. This value is in good agreement with our previous results for the intrinsic damping of permalloy [26] and the literature cited therein.

To investigate different damping contributions, we also determine the full width at half-maximum $\Delta H(f)$ of the transmission via Lorentzian fits. This width is displayed for different high-frequency field strength in Fig. 2(c). For small high-frequency field strengths up to $\mu_{0} H_{\mathrm{rf}}=0.47 \mathrm{mT}$ the linewidth $\Delta H(f)$ depends almost linearly on the frequency $f$ and therefore indicates a linear Gilbert-damping regime without significant two-magnon damping contribution [28]. For high-frequency field strengths above $0.47 \mathrm{mT}$, an increasing quadratic contribution is visible.

Figure 3 shows FMR spectra $\Delta T$ for an excitation field of $\mu_{0} H_{\mathrm{rf}}=0.19 \mathrm{mT}$ for both sample types, respectively. A comparison shows that the Py/Pt sample exhibits a broader peak shape than the Py/- sample, attributed to the aforementioned increased damping by spin pumping. The slight shift of the resonance position results from the preparation process, since the Py/Pt samples are cleaned additionally via rf argon-plasma etching before the platinum is deposited. Hence the permalloy thickness is reduced.

In order to investigate the influence of the rf excitation field amplitude on the FMR mode multiple FMR measurements are performed. For this purpose the strength of the high-frequency excitation field is varied from $0.06 \mathrm{mT}$ to $1.89 \mathrm{mT}$. To be able to compare these FMR measurements more directly with the voltage measurements shown later, we now switch the reference method from a reference field to a reference frequency. For this measurement technique again the static 
magnetic field is varied, while for every field step, the averaged transmission through the waveguide is measured for a measurement frequency $f_{\text {meas }}$ and a reference frequency $f_{\text {ref. }}$. Here the reference frequency is always $f_{\text {ref }}=2 \mathrm{GHz}$, since this frequency does not cross the FMR mode. The transmission through the waveguide is then determined as $\Delta T_{\mathrm{f}}=T\left(f_{\text {meas }}\right)-T\left(f_{\text {ref }}\right)$. Note, that for the field reference method the reference signal is obtained using a static magnetic field of $\mu_{0} H=90 \mathrm{mT}$ that is aligned along the $y$ direction. Equivalent measurements for both reference methods are shown in Fig. 3. For the frequency reference method additional offsets remain due to the frequency-dependent damping of the waveguide. If these offsets are removed, the transmission signals are similar. As mentioned before, the frequencydependent damping of the waveguide is taken into account in the estimation of the high-frequency fields.

In Fig. 4(a) a detailed analysis of the transmission signal $\Delta T_{\mathrm{f}}$ in dependence of different high-frequency field strengths
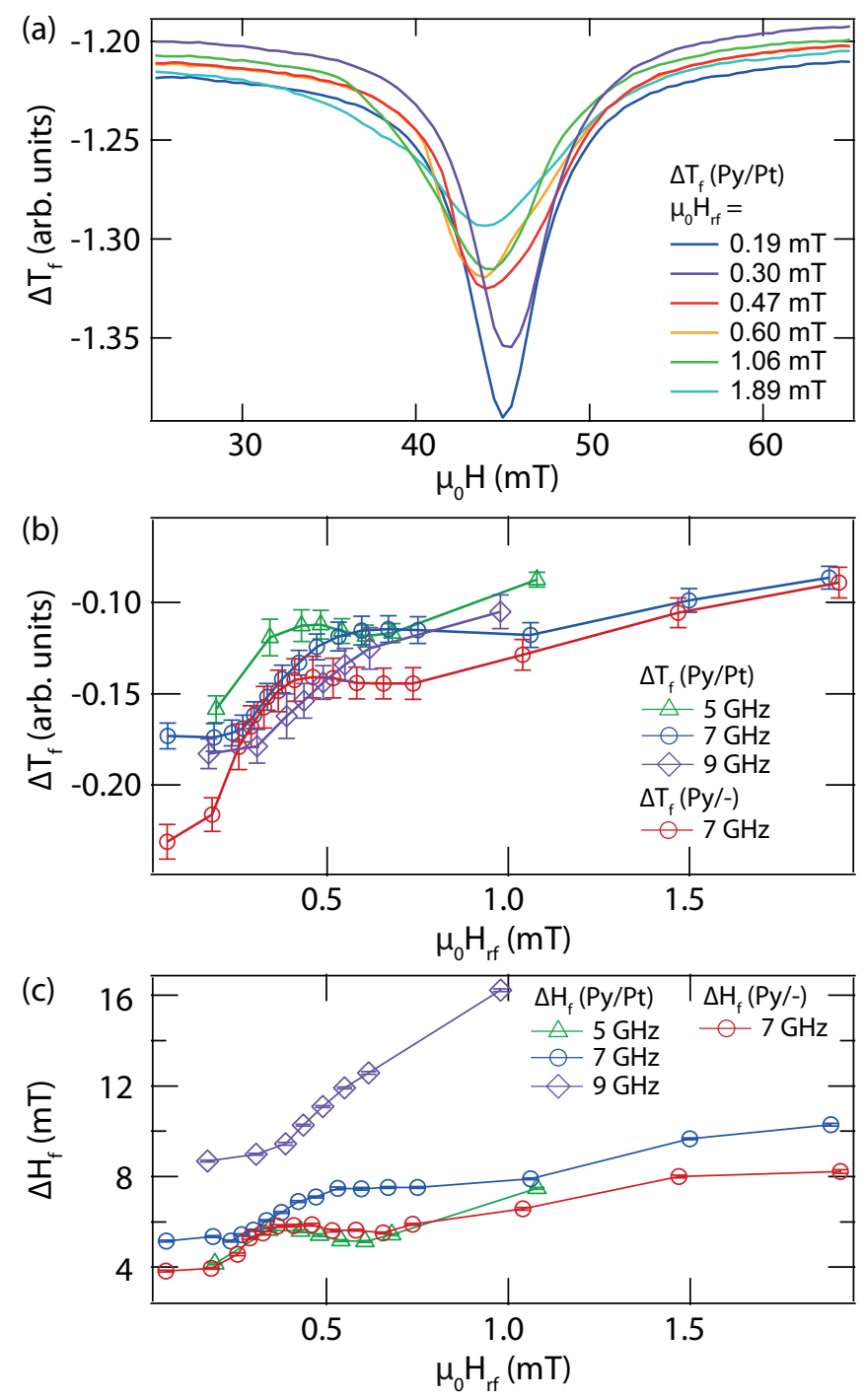

FIG. 4. (a) Field-dependent transmission spectra for a constant frequency of $7 \mathrm{GHz}$ and various high-frequency field strengths. Evaluation of the transmission peak heights (b) and full widths at half-maximum (c). for the Py/Pt sample is shown. The aforementioned change between the linear and nonlinear (quadratic) damping regime for $\mu_{0} H_{\mathrm{rf}}=0.47 \mathrm{mT}$, cf. Fig. 2(c), corresponds to the beginning of the plateau of the transmission amplitudes, displayed in Fig. 4(b). The dependence of the peak width at half-height $\Delta H_{\mathrm{f}, \mathrm{Py} / \mathrm{Pt}}$ of a bilayer sample on the excitation strength is shown in Fig. 4(c) for three excitation frequencies. Since the peak widths at half-height behave similarly for both investigated sample types and both field branches, only the values of the positive field branch for the Py/Pt sample are displayed. An increase of the excitation frequency, as well as an increase of the excitation field leads to an increased peak width at half-height, see also Fig. 2(c). However, the values of $\Delta H_{\mathrm{f}}$ for high excitation fields need to be treated carefully, because the transmission curves broaden and, thus, the peak shape is less Lorentzian.

For the spin-transport measurements again a highfrequency field $H_{\mathrm{rf}}$ is used to excite the resonant precession of the permalloy element. Spin pumping occurs and the voltage drop from the resulting charge current in the Pt layer, generated by the inverse spin-Hall effect, is measured by a voltmeter, connected to the sample via rf probes that are positioned on the leads. The external static magnetic field is varied in steps of $0.5 \mathrm{mT}$. The measurement frequency is chosen so as to cross the FMR mode twice, cf. Fig. 2(a), once for negative and once for positive values of the external magnetic field. The reference frequency is below the FMR mode and crosses no resonance. For the displayed measurements $f_{\text {ref }}=2 \mathrm{GHz}$. For every field step the transmission through the waveguide is measured ten times for $f_{\text {meas }}$ and $f_{\text {ref }}$. The averaged difference $\Delta T_{\mathrm{f}}$ of these values is almost free of contributions that are not induced by FMR [29]. Afterwards the voltage measurements are performed similarly for the two frequencies, but repeated 100 times for every field step, so that the averaged voltage difference is given by

$$
\Delta V=\frac{\Sigma_{i}\left[V_{i}\left(f_{\text {meas }}\right)-V_{i}\left(f_{\text {ref }}\right)\right]}{100} .
$$

Note, that the transmission data is generated by the whole ensemble of equal permalloy rectangles, while the voltage signal is obtained from the centered permalloy rectangle connected to the leads. It is known from the literature that the perpendicular orientation between the static magnetic field that is parallel to the easy axis of the permalloy rectangles and the high-frequency field in combination with the position of the voltage contacts allow for an optimum ratio between symmetric and asymmetric voltage contributions $[9,11,13,14,16,17]$. Exemplary transport measurements can be seen in Figs. 5(a) and 5(b) for both field-sweep directions. The light blue line indicates the transmission $\Delta T_{\mathrm{f}}$, the blue circles are the voltage data $\Delta V$, and the black line shows a Lorentzian fit of each peak in the voltage data. The voltage peaks exhibit a sign reversal on magnetic field reversal. This is a typical feature of the inverse spin-Hall effect [15], since the magnetization, and, thus, the spin polarization, switches at $\pm 3 \mathrm{mT}$, cf. Eq. (2). Simultaneously, the flow direction of the spin current stays the same, cf. Eq. (1). The sign reversal is also valid for the smaller voltage peaks that are attributed to the first SW-S mode. It can be seen that the external field values for the minimal transmission of the FMR signal and 

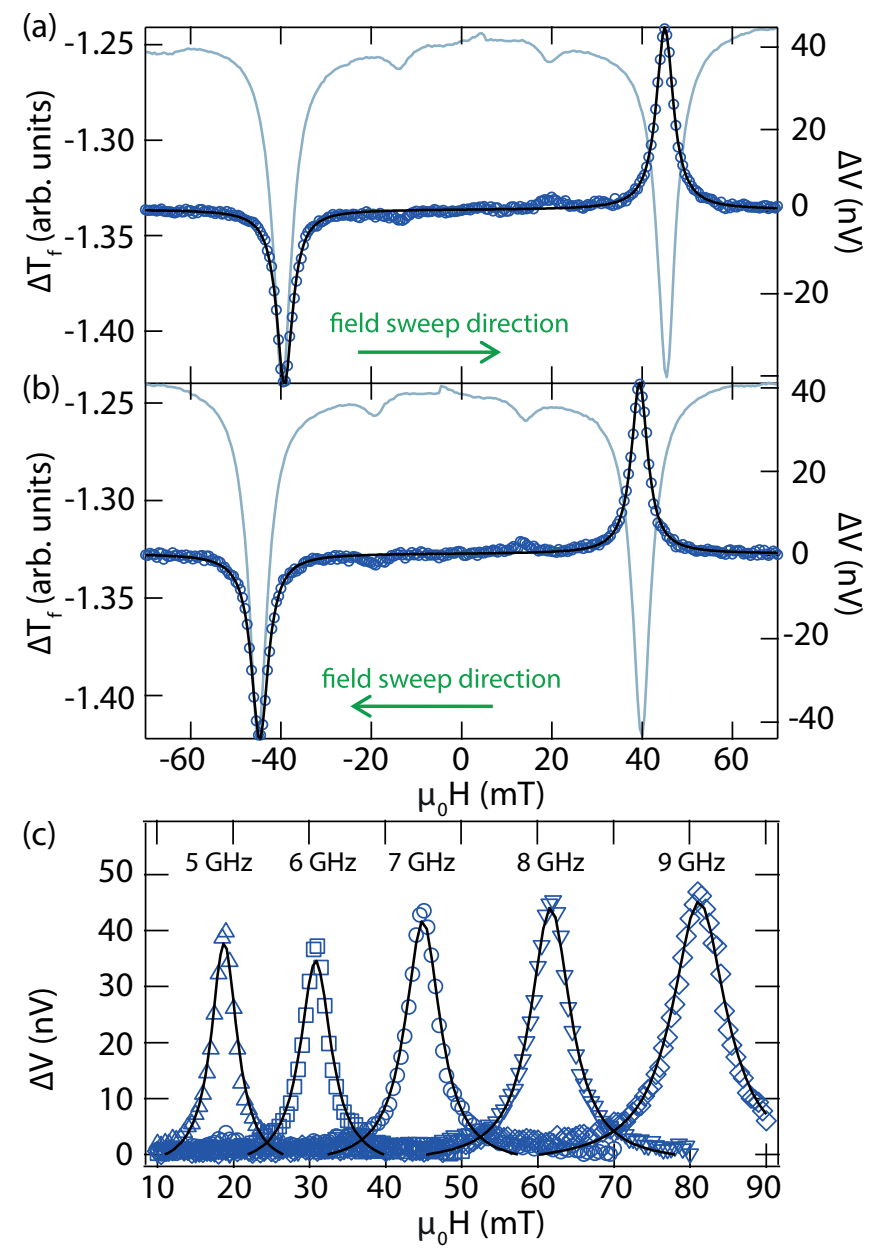

FIG. 5. Transmitted excitation power $\Delta T_{\mathrm{f}}$ and inverse spin-Hall effect voltage $\Delta V$ for (a) an up and (b) a down sweep of the external static magnetic field $\mu_{0} \mathrm{H}$ for a constant frequency of $7 \mathrm{GHz}$ and a high-frequency field amplitude of $0.19 \mathrm{mT}$. The transmission is plotted as light blue line, the voltage data are the blue circles and the black line is a Lorentzian fit. (c) Voltage measurements for different excitation frequencies of the high-frequency field with a constant amplitude of $0.19 \mathrm{mT}$.

for the extrema of the voltage are matching very well. Small deviations between the resonance positions of the positive in comparison with the negative field branch can be attributed to the hysteresis of the magnets that generate the external magnetic field $H$. To minimize such inaccuracies due to the setup the total mean value for the voltage peak height $\Delta V_{\max }$ and the resonant field $\mu_{0} H_{\text {res }}$ can be determined as the absolute mean value of the four peaks: $\Delta V_{\max }=(43.19 \pm 1.69) \mathrm{nV}$ and $\mu_{0} H_{\text {res }}=(42.08 \pm 3.21) \mathrm{mT}$. Since there are no remarkable differences between the peaks, from now on field up sweeps and the positive branches are shown.

The inverse spin Hall voltage generated in the platinum layer can be estimated as follows [13]:

$$
V_{\mathrm{ISHE}}=\frac{\Theta_{\mathrm{ISHE}} \lambda_{\mathrm{sd}} e f_{\text {meas }} P \sin ^{2}(\theta) g_{\mathrm{eff}}^{\uparrow \downarrow} l}{\sigma_{\mathrm{Pt}} t_{\mathrm{Pt}}+\sigma_{\mathrm{Py}} t_{\mathrm{Py}}} \tanh \left(\frac{t_{\mathrm{Pt}}}{2 \lambda_{\mathrm{sd}}}\right),
$$

with the elementary charge $e$. The spin Hall angle $\Theta_{\text {ISHE }}$ and the spin-diffusion length of platinum $\lambda_{\text {sd }}$ are vividly discussed and range between $\Theta_{\text {ISHE }}=0.013$ and 0.33 and $\lambda_{\text {sd }}=1.2 \mathrm{~nm}$ and $10 \mathrm{~nm}[13,30-33]$. For an excitation field similar to $0.22 \mathrm{mT}$ the cone angle of the magnetization precession is $[20,21] \theta \approx 1.4^{\circ}$. The ellipticity correction factor $P \approx 0.7$ for $f_{\text {meas }}=7 \mathrm{GHz}$ considers the elliptical trajectory of the magnetization precession [13]. The charge conductivities are $\sigma_{\mathrm{Pt}}=(2.4 \pm 0.2) \times 10^{6}(\Omega \mathrm{m})^{-1}$ for platinum[13] and $\sigma_{\mathrm{Py}}=$ $3.6 \times 10^{6}(\Omega \mathrm{m})^{-1}$ for permalloy [26]. The respective thicknesses are $t_{\mathrm{Pt}}=15 \mathrm{~nm}$ and $t_{\mathrm{Py}}=30 \mathrm{~nm}$. The effective spinmixing conductance $g_{\text {eff }}^{\uparrow \downarrow}$ includes a possible spin backflow into the ferromagnet that reduces the spin-pumping current injected into the platinum layer [13,34],

$$
g_{\mathrm{eff}}^{\uparrow \downarrow}=\frac{4 \pi M_{\mathrm{s}} t_{\mathrm{Py}}}{g \mu_{B}}\left(\alpha_{\mathrm{Py} / \mathrm{Pt}}-\alpha_{\mathrm{Py} /-}\right),
$$

whereby $g=2$ is the Landé factor, and $\mu_{B}=9.274 \times$ $10^{-24} \mathrm{~J} \mathrm{~T}^{-1}$ is the Bohr magneton. The effective spin-mixing conductance is calculated using information from the FMR measurements, for an excitation field of $0.19 \mathrm{mT}$, that is the saturation magnetization $M_{\mathrm{s}}=835 \mathrm{kA} \mathrm{m}^{-1}$, and the damping parameters $\alpha_{\mathrm{Py} / \mathrm{Pt}}=0.0069$ and $\alpha_{\mathrm{Py} /-}=0.0046$. For $\mu_{0} H_{\mathrm{rf}}=$ $0.19 \mathrm{mT}$ the effective spin-mixing conductance yields $g_{\text {eff }}^{\uparrow \downarrow}=$ $3.90 \times 10^{19} \mathrm{~m}^{-2}$. This value is slightly higher than recently reported values $[13,14,34,35]$ that range between $g_{\text {eff }}^{\uparrow \downarrow}=$ $1.5 \times 10^{19} \mathrm{~m}^{-2}$ and $3.0 \times 10^{19} \mathrm{~m}^{-2}$. But a direct comparison is difficult, since the value of the effective spin-mixing conductance depends on the high-frequency excitation field strength, since the linewidth and, thus, the damping of the Py/sample and the Py/Pt sample varies, compare Fig. 4(c). The ISHE voltage, generated in between the two leads, connected to the edges of the platinum strip, with a distance $l=1340 \mathrm{~nm}$, can then be predicted using the different parameter sets for the spin Hall angle and the spin-diffusion length [30-33] between $V_{\text {ISHE }}=14 \mathrm{nV}$ and $V_{\text {ISHE }}=97 \mathrm{nV}$. These values are in good agreement with the overall mean value of the measured maximal voltage $\Delta V_{\max }=(43.19 \pm 1.69) \mathrm{nV}$, determined from the measurements displayed in Figs. 5(a) and 5(b) for a frequency of $7 \mathrm{GHz}$ and an excitation field of $0.19 \mathrm{mT}$.

In order to investigate the dependency between the excitation parameters and the voltage that is generated by the ISHE, the excitation frequency and the high-frequency field amplitude $H_{\mathrm{rf}}$ are varied. Transport measurements for frequencies between 5 and $9 \mathrm{GHz}$ are shown in Fig. 5(c). As expected, due to the course of the FMR mode that is governed by the Kittel formula, the resonance position increases for increasing excitation frequency $f_{\text {meas }}$. The voltage peaks exhibit a Lorentzian shape.

In Fig. 6 transport measurements for different highfrequency excitation field amplitudes are displayed for a constant frequency of $7 \mathrm{GHz}$. For excitation fields smaller than $\mu_{0} H_{\mathrm{rf}}=0.47 \mathrm{mT}$ the Hall voltage obeys a Lorentzian shape and the maximal peak height $\Delta V_{\max }$, shown in Fig. 7(a), increases quadratically with $\mu_{0} H_{\mathrm{rf}}$. This correlation occurs because the voltage depends approximately quadratically on the cone angle $\theta$ that is proportional to the high-frequency excitation field strength $[11,13,14,36,37]$. By increasing the excitation field, the voltage peak shifts to smaller external fields and a slight asymmetry in the slope of the flanks appears. This effect is discussed in Sec. V. Figure 6(b) shows the 


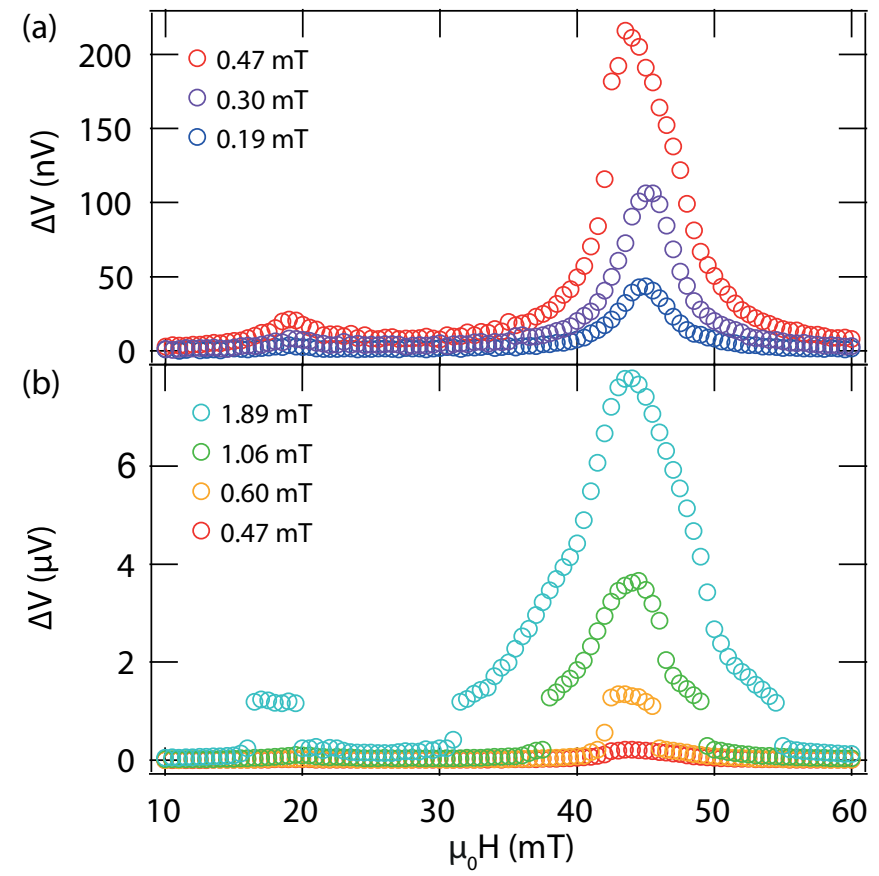

FIG. 6. Inverse spin Hall voltage $\Delta V$ versus static magnetic field $H$ for low high-frequency field amplitudes $H_{\mathrm{rf}}$ (a) and high amplitudes (b) with a constant excitation frequency of $7 \mathrm{GHz}$.

voltage for high-frequency excitation fields $\mu_{0} H_{\mathrm{rf}} \geqslant 0.47 \mathrm{mT}$. At $\mu_{0} H_{\mathrm{rf}}=0.60 \mathrm{mT}$ the system jumps into a new regime. The maximal peak height abruptly increases from $215 \mathrm{nV}$ to $1.2 \mu \mathrm{V}$. At the same time the voltage peaks broaden and deviate from the Lorentzian shape. In addition, all voltage peaks in Fig. 6(b) exhibit a gap in the voltage data in between approximately $200 \mathrm{nV}$ and $1 \mu \mathrm{V}$. The maximal ISHE voltages for the positive and negative fields $+\Delta V_{\max }$ and $-\Delta V_{\max }$ for various high-frequency excitation field amplitudes are shown in Fig. 7(b). Here the two regimes with the transition at $\mu_{0} H_{\mathrm{rf}}=0.60 \mathrm{mT}$ are well visible. The transfer between the two regimes is highly reproducible and corresponds with the change between linear and nonlinear regime in the FMR measurements. To gain a deeper understanding of this transition the variation of the high-frequency field amplitude is repeated for different excitation frequencies, shown in Fig. 7(c). These measurements show that the transition between the two regimes is close to invariant for different frequencies. The negative field branch is displayed in the Supplemental Material [38]. We have also performed the same measurements on a sample with larger permalloy width and on a sample with nonperpendicularly oriented edges, see the Supplemental Material [38]. An evaluation of the voltage maxima revealed no qualitative changes, only a slight shift of the voltage surge towards smaller high-frequency fields for the sample with the increased width and towards larger high-frequency fields for the sample with nonperpendicularly oriented edges occurs. Indeed, ISHE voltages up to a few microvolts have been reported before [9-17]. However, the lateral dimensions of the bilayers used there are typically one or two orders of magnitude larger than the ones used here. Consequently larger ISHE voltage signals are measured, since the length $l$ between the voltage contacts is proportional to the
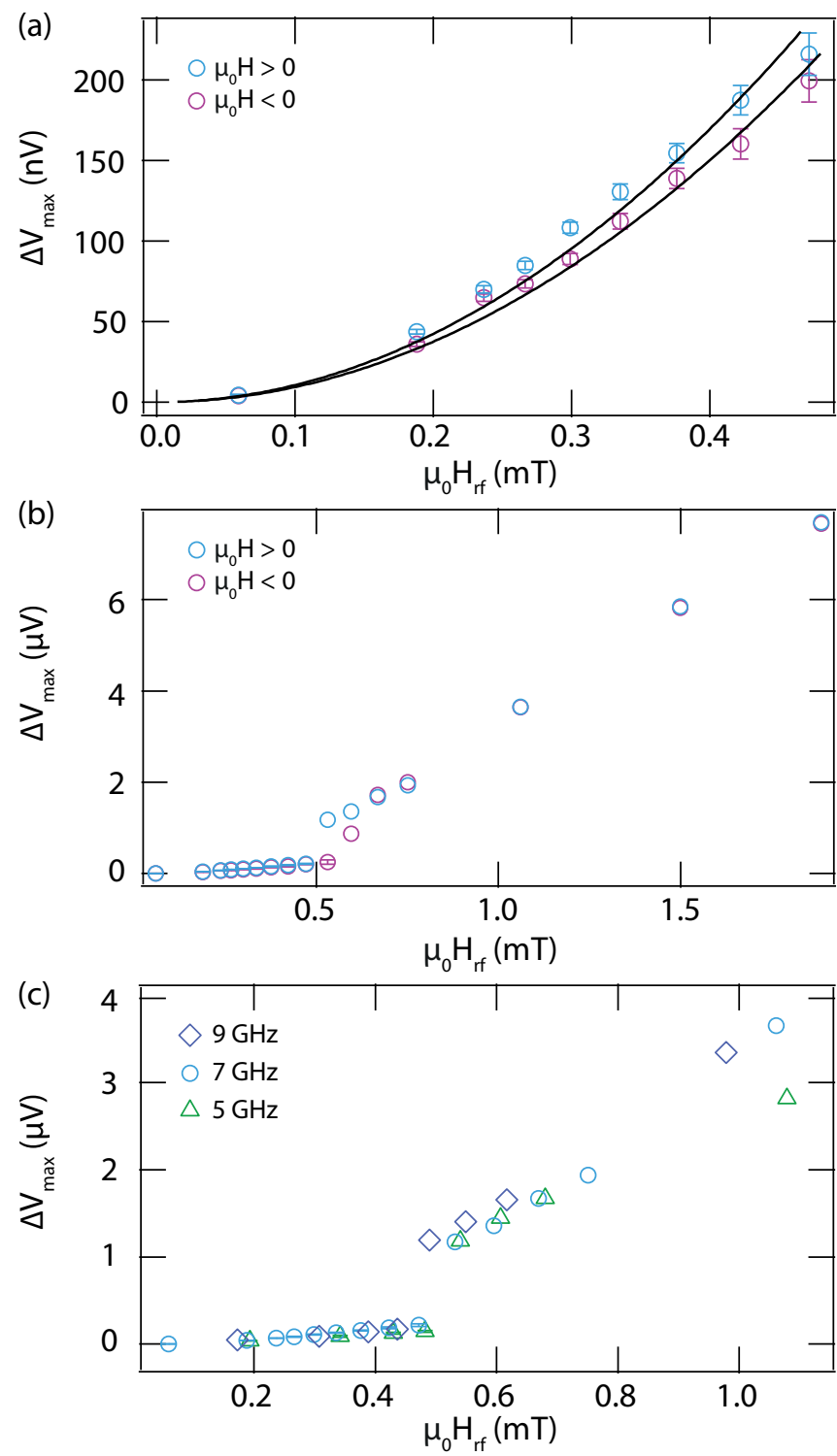

FIG. 7. ISHE voltage peak amplitudes $\pm \Delta V_{\max }$ versus highfrequency field amplitude $H_{\mathrm{rf}}$ for positive (cyan) and negative (fuchsia) field branch and a constant excitation frequency of $7 \mathrm{GHz}$. Note, that in (a) only low high-frequency field amplitudes up to $\mu_{0} H_{\mathrm{rf}}=0.47 \mathrm{mT}$ are displayed, while (b) shows amplitudes up to $1.89 \mathrm{mT}$. In (c) the high-frequency field dependence for additional frequencies is supplemented for the positive field branch.

ISHE voltage, cf. Eq. (6). For example in the publication of Mosendz et al. [13], the maximal obtained current density is $2 \times 10^{5} \mathrm{~A} \mathrm{~m}^{-2}$. For our samples it is up to $2 \times 10^{7} \mathrm{~A} \mathrm{~m}^{-2}$ for a high-frequency field amplitude of $1.89 \mathrm{mT}$. Note, that in our measurements higher excitation field strength can be reached, because the bilayer microstructures are placed on top of the only $3.6 \mu \mathrm{m}$ wide signal lead of the waveguide.

\section{MICROMAGNETIC SIMULATIONS}

In order to identify the cause of the abrupt increase in the measured voltage at a threshold field we performed micromagnetic simulations using MicroMagnum [39,40]. A 
8- $\mu \mathrm{m}$-long, $2-\mu \mathrm{m}$-wide, and 30-nm-thick permalloy element is simulated in a static $38 \mathrm{mT}$ magnetic field along the $x$ axis. Perpendicular to the static field an rf in-plane excitation field at $7 \mathrm{GHz}$ is applied along the $y$ axis similar to the measurements presented in Sec. III. For excitation field amplitudes from $0.01 \mathrm{mT}$ to $1 \mathrm{mT}$ the magnetization dynamics are recorded by increasing the amplitude by $0.01 \mathrm{mT} / \mathrm{ns}$ during the simulation. The voltage measurements are most sensitive to the region in between the copper leads and from Eq. (6) the voltage is proportional to the $\sin ^{2}(\theta)$ where $\theta$ is the cone angle of the magnetization. Therefore, we calculated the cone angle for every simulation cell within a $1.6-\mu \mathrm{m}$-long and $1.2-\mu \mathrm{m}$-wide center region of the permalloy element. Because the magnetization precession is elliptical we consider cone
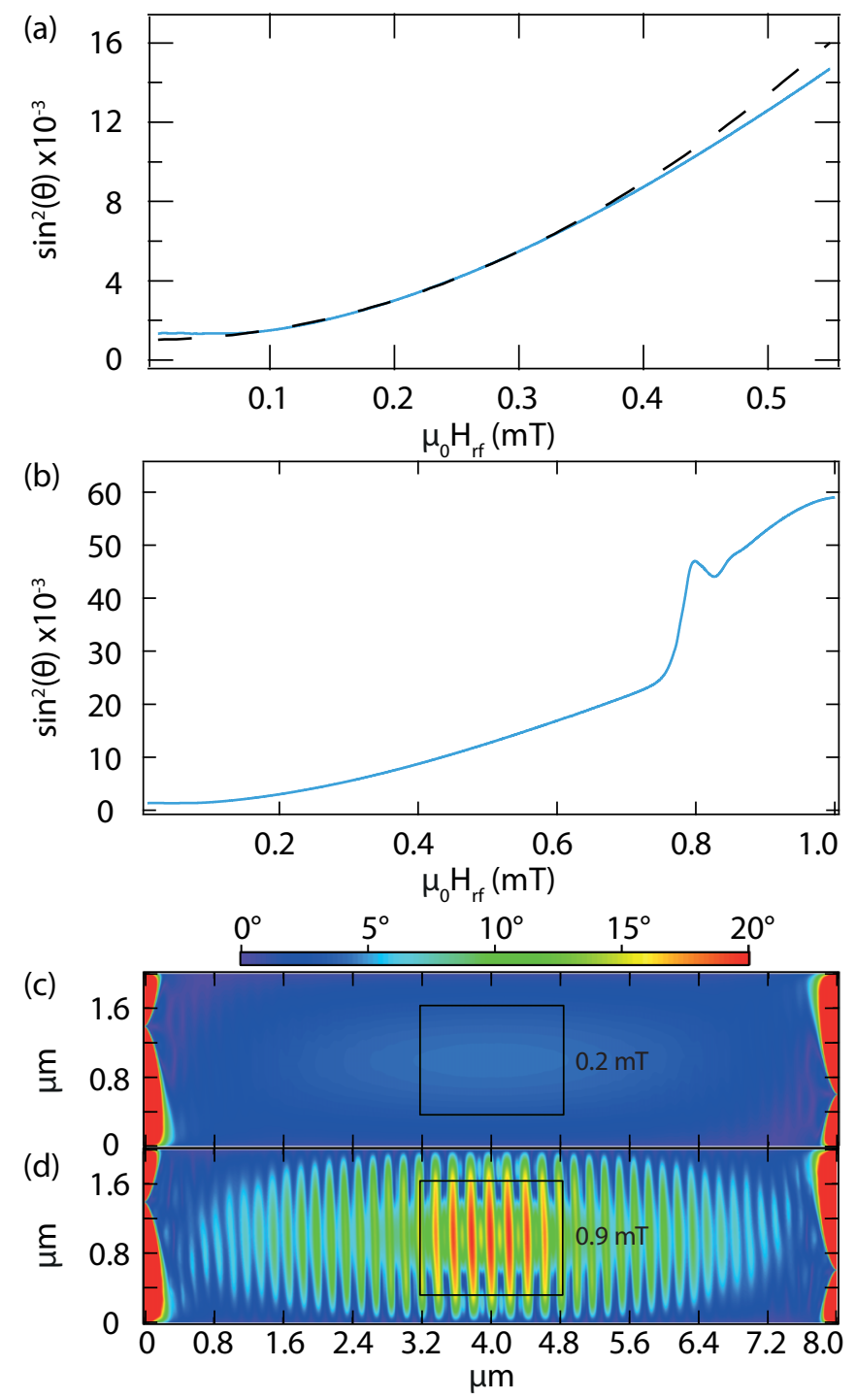

FIG. 8. Micromagnetic simulations of the permalloy rectangles' magnetization dynamics. Shown is the average $\sin ^{2}(\theta)$ in the center region of the permalloy rectangle. It is displayed for an excitation field up to $0.55 \mathrm{mT}$ in (a) and up to $1.0 \mathrm{mT}$ in (b). In (c) and (d) exemplary plots of the local cone angle $\theta$ for excitation field strengths of 0.2 and $0.9 \mathrm{mT}$ at $7 \mathrm{GHz}$ are shown. The black rectangles mark the region of the permalloy rectangles between the voltage leads that are used for the calculation of the average $\sin ^{2}(\theta)$.

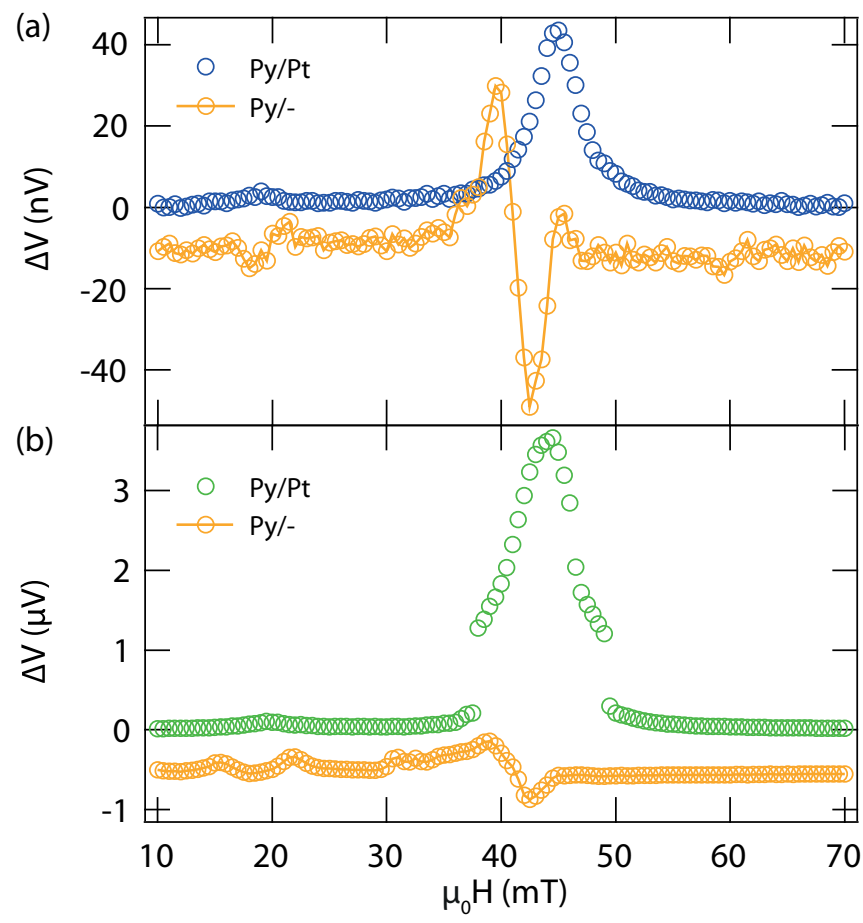

FIG. 9. (a) Voltage measurements of a Py/Pt (blue circles) and a Py/- (yellow circles and line) sample for an rf excitation field of $0.19 \mathrm{mT}$. (b) Voltage measurements of a Py/Pt (green circles) and a Py/- (yellow circles and line) sample for an rf excitation field of 1.06 $\mathrm{mT}$. All measurements are performed with an excitation frequency of $7 \mathrm{GHz}$.

angle averages of full excitation periods. The averaged $\sin ^{2}(\theta)$ within the region of interest for excitation amplitudes smaller than $0.55 \mathrm{mT}$ is shown in Fig. 8(a). As expected it increases quadratically with increasing excitation power, comparable to the measured voltage in Fig. 7(a). Figure 8(b) shows the averaged $\sin ^{2}(\theta)$ for excitation amplitudes up to $1 \mathrm{mT}$. Above $0.3 \mathrm{mT}$ the increase becomes linear until an abrupt surge is found for excitation amplitudes larger than $0.77 \mathrm{mT}$. This qualitatively resembles the measured behavior shown in Fig. 7(c). The small quantitative differences can be attributed to the differences between simulation and measurement, e.g., missing surface roughness in the simulation. With the surge a change in the excited magnetization pattern is observed. Below $0.77 \mathrm{mT}$ the center region exhibits an almost uniform precession of the magnetization corresponding to the FMR mode, as shown in an exemplary cone angle plot for an excitation strength of $0.2 \mathrm{mT}$ in Fig. 8(c). With increasing field amplitude the uniform gyration gains amplitude until $0.77 \mathrm{mT}$, where a superposed spin-wave mode with a magnetostatic backward volume character [24,41,42] emerges in the center of the permalloy elements, see Fig. 8(d) for an excitation of $0.9 \mathrm{mT}$. The identification as a spin wave and not as a pure amplitude modulation of the excited magnetization is verified by analysis of the phase relation (not shown).

\section{DISCUSSION}

In similar experiments investigating permalloy/platinum bilayers, other possible contributions to the measured voltage described in Sec. III are reported. The most important 
contributions are analyzed in the following with respect to their importance for our measurements, especially concerning their potential to cause the two voltage regimes. One of these contributions is the spin-rectification effect [43] in the permalloy rectangle that can interfere with the voltage generated by the inverse spin-Hall effect $[9,11,13,14,16,44,45]$. In order to analyze these voltage contributions, transport measurements with a Py/- sample (without the platinum layer) are performed. For this sample the leads are directly deposited on top of the permalloy rectangle. The measured voltage can then be generated via the spin-rectification effect (SRE) due to the anisotropic magnetoresistance $[13,14,16,17,43]$ or the anomalous Hall effect $[9,11,16,43]$.

A direct comparison between the two sample types is shown for two excitation field amplitudes in Fig. 9. The voltages generated by the SRE (yellow circles and line) in the Py/- samples are antisymmetric with respect to the resonance position and exhibit a small offset voltage. In contrast, the voltage peaks of the $\mathrm{Py} / \mathrm{Pt}$ samples are symmetric (green and blue circles). For the geometry of the voltage contacts of our bilayer samples no voltage asymmetry is expected, because the permalloy has no direct contact with the voltage leads. For increasing excitation fields, as shown in Fig. 9(b), the measured voltage for both sample types increases. However, for the Py/- sample no abrupt increase of the voltage is observed and it is therefore clearly smaller in comparison to the symmetric ISHE contribution. Nonetheless small antisymmetric contributions may superpose with the symmetric voltage generated by the inverse spin-Hall effect $[13,14,16]$. Since the measurements shown in Sec. III are symmetric with respect to the resonance position for small excitation fields, a significant antisymmetric contribution can be excluded.

Another possible voltage contribution that should be discussed is the influence of self-oscillation of the magnetization [46] and spin-torque effects [47,48]. A self-oscillation of the magnetization can be caused by an electrical dc current that is sent directly through the sample. Thereby current densities of minimally $3 \times 10^{12} \mathrm{~A} \mathrm{~m}^{-2}$ are needed in a platinum layer to overcome the critical field and excite an auto-oscillation [46]. It is unlikely that these current densities are reached in our structures via inductive coupling. Spin-torque effects generating a dc voltage have been reported for Py/Pt bilayer samples where an rf charge current is applied to the platinum [48] or to the bilayer [47]. In principle it is possible that the high-frequency field generates an oscillating high-frequency current in the platinum layer via induction. This in return could cause a spin-torque contribution in the voltage and FMR measurements. However, we do not consider these cascaded secondary effects to cause the observed voltage surge.

Further differences between predictions and measurements may be caused by the used approximations. The concept of ferromagnetic resonance considers a linear damping of the magnetic system following the Landau-Lifshitz-Gilbert equation. Especially for the description of spin currents, approximations for sufficiently small cone angles $\theta$ are used. However, an increase of the excitation field leads to an increase of the cone angle, as can be seen in Fig. 8(d), where locally angles of up to $20^{\circ}$ are reached. Therewith, nonlinear FMR responses and foldover effects also known as
Suhl instability processes need to be considered [18,27,49,50]. In the FMR measurements the transition between linear and nonlinear damping can be identified as an increasing derivation from the linear relation between the peak width at half-maximum $\Delta H$ and the excitation frequency, cf. Fig. 2(c). This change correlates with the beginning of the plateau of the heights of the transmission maxima, see Fig. 4(b). Recent studies by Bauer et al. show that in permalloy nonlinear magnetization dynamics due to large high-frequency field amplitudes leads to nonlinear parametric spin-wave excitation [18]. In this work the magnon density in dependence on the high-frequency excitation field strength has been determined. For an increasing excitation strength at first the number of uniformly precessing magnons rises until a threshold value is reached. At this point also nonuniform magnons with $k \neq 0$ are increasingly excited and the number of uniform magnons saturates. In comparison with our measurements the magnon density calculated by Bauer et al. [18] can be identified to be inversely proportional to the height of our transmission maxima. Using this comparison the beginning of the plateau of the transmission maxima and, thus, the nonlinearities in our measurements can be identified as the high-frequency excitation field strength, where the excitation of uniform magnons saturates and nonuniform magnons with $k \neq 0$ are increasingly excited [18]. An excitation of nonuniform magnons can also be seen at high excitation field strength in our micromagnetic simulations. Since the beginning of the plateau of the transmission maxima corresponds to the occurrence of the voltage surge we deduce the two voltage regimes to be caused by nonlinear spin-wave excitation.

In summary, the voltage generated in the microstructured $\mathrm{Py} / \mathrm{Pt}$ samples is dominated by the inverse spin-Hall effect. By increasing the excitation field strength, an abrupt and frequency independent surge of one order of magnitude of the ISHE voltage is observed. This transition is reproducible and has not been reported so far. It correlates with the occurrence of nonlinear damping in the FMR measurements. In the high excitation field regime current densities of up to $2 \times 10^{7} \mathrm{~A} \mathrm{~m}^{-2}$ are reached in our sample, which is two orders of magnitude larger than in conventional samples. Micromagnetic simulations in combination with recent publications and our FMR measurements suggest that the nonlinear voltage surge results from a superposition of the uniform mode with nonuniform spin-wave modes. The spin-rectification effect in the permalloy rectangle as well as self-oscillation of the magnetization and spin-torque effects are not responsible for the strong enhancement of the voltage. The presence of two distinct regimes in combination with high voltage-signals of up to a few microvolts in a micron-sized device opens new possibilities in the development of future spintronic devices.

\section{ACKNOWLEDGMENTS}

We thank Ulrich Merkt, Christian Swoboda, and Lars Bocklage for fruitful discussions. We would also like to thank the two anonymous referees for a very fruitful scientific discussion, which motivated additional measurements and clearly helped to further improve our manuscript. Financial support by the Deutsche Forschungsgemeinschaft via Sonderforschungsbereich 688 and via Graduiertenkolleg 1286 is gratefully acknowledged. 
[1] A. Brataas, Y. Tserkovnyak, G. E. W. Bauer, and B. I. Halperin, Phys. Rev. B 66, 060404 (2002).

[2] R. Urban, G. Woltersdorf, and B. Heinrich, Phys. Rev. Lett. 87, 217204 (2001).

[3] S. Mizukami, Y. Ando, and T. Miyazaki, Phys. Rev. B 66, 104413 (2002).

[4] Y. Tserkovnyak, A. Brataas, and G. E. W. Bauer, Phys. Rev. Lett. 88, 117601 (2002).

[5] A. Brataas, A. Kent, and H. Ohno, Nat. Mater. 11, 372 (2012).

[6] S. Dushenko, M. Koike, Y. Ando, T. Shinjo, M. Myronov, and M. Shiraishi, Phys. Rev. Lett. 114, 196602 (2015).

[7] M. V. Costache, S. M. Watts, C. H. van der Wal, and B. J. van Wees, Phys. Rev. B 78, 064423 (2008).

[8] J. C. Rojas Sánchez, L. Vila, G. Desfonds, S. Gambarelli, J. P. Attané, J. M. De Teresa, C. Magén, and A. Fert, Nat. Commun. 4, 2944 (2013).

[9] E. Saitoh, M. Ueda, H. Miyajima, and G. Tatara, Appl. Phys. Lett. 88, 182509 (2006).

[10] H. Nakayama, K. Ando, K. Harii, T. Yoshino, R. Takahashi, Y. Kajiwara, K. Uchida, Y. Fujikawa, and E. Saitoh, Phys. Rev. B 85, 144408 (2012).

[11] H. Y. Inoue, K. Harii, K. Ando, K. Sasage, and E. Saitoh, J. Appl. Phys. 102, 083915 (2007).

[12] K. Ando, Y. Kajiwara, S. Takahashi, S. Maekawa, K. Takemoto, M. Takatsu, and E. Saitoh, Phys. Rev. B 78, 014413 (2008).

[13] O. Mosendz, V. Vlaminck, J. E. Pearson, F. Y. Fradin, G. E. W. Bauer, S. D. Bader, and A. Hoffmann, Phys. Rev. B 82, 214403 (2010).

[14] A. Azevedo, L. H. Vilela-Leão, R. L. Rodríguez-Suárez, A. F. Lacerda Santos, and S. M. Rezende, Phys. Rev. B 83, 144402 (2011).

[15] F. D. Czeschka, L. Dreher, M. S. Brandt, M. Weiler, M. Althammer, I.-M. Imort, G. Reiss, A. Thomas, W. Schoch, W. Limmer, H. Huebl, R. Gross, and S. T. B. Goennenwein, Phys. Rev. Lett. 107, 046601 (2011).

[16] E. T. Papaioannou, P. Fuhrmann, M. B. Jungfleisch, T. Brächer, P. Pirro, V. Lauer, J. Lösch, and B. Hillebrands, Appl. Phys. Lett. 103, 162401 (2013).

[17] M. Obstbaum, M. Härtinger, H. G. Bauer, T. Meier, F. Swientek, C. H. Back, and G. Woltersdorf, Phys. Rev. B 89, 060407 (2014).

[18] H. G. Bauer, P. Majchrak, T. Kachel, C. H. Back, and G. Woltersdorf, Nat. Commun. 6, 8274 (2015).

[19] T. J. Silva, C. S. Lee, T. M. Crawford, and C. T. Rogers, J. Appl. Phys. 85, 7849 (1999).

[20] N. Kuhlmann, Dissertation, Universität Hamburg, 2014, p. 95.

[21] N. Kuhlmann, A. Vogel, and G. Meier, Phys. Rev. B 85, 014410 (2012).

[22] C. Swoboda, N. Kuhlmann, M. Martens, A. Vogel, and G. Meier, J. Appl. Phys. 114, 043905 (2013).

[23] R. W. Damon and J. R. Eshbach, J. Phys. Chem. Solids 19, 308 (1961).

[24] J. Jorzick, S. O. Demokritov, B. Hillebrands, M. Bailleul, C. Fermon, K. Y. Guslienko, A. N. Slavin, D. V. Berkov, and N. L. Gorn, Phys. Rev. Lett. 88, 047204 (2002).

[25] S. S. Kalarickal, P. Krivosik, M. Wu, C. E. Patton, M. L. Schneider, P. Kabos, T. J. Silva, and J. P. Nibarger, J. Appl. Phys. 99, 093909 (2006).
[26] G. Nahwold, J. Scholtyssek, S. Motl-Ziegler, O. Albrecht, U. Merkt, and G. Meier, J. Appl. Phys. 108, 013907 (2010).

[27] Y. S. Gui, A. Wirthmann, N. Mecking, and C.-M. Hu, Phys. Rev. B 80, 060402 (2009).

[28] K. Lenz, H. Wende, W. Kuch, K. Baberschke, K. Nagy, and A. Jánossy, Phys. Rev. B 73, 144424 (2006).

[29] N. Kuhlmann, C. Swoboda, A. Vogel, T. Matsuyama, and G. Meier, Phys. Rev. B 87, 104409 (2013).

[30] M. Althammer, S. Meyer, H. Nakayama, M. Schreier, S. Altmannshofer, M. Weiler, H. Huebl, S. Geprägs, M. Opel, R. Gross, D. Meier, C. Klewe, T. Kuschel, J.-M. Schmalhorst, G. Reiss, L. Shen, A. Gupta, Y.-T. Chen, G. E. W. Bauer, E. Saitoh, and S. T. B. Goennenwein, Phys. Rev. B 87, 224401 (2013).

[31] W. Zhang, V. Vlaminck, J. E. Pearson, R. Divan, S. D. Bader, and A. Hoffmann, Appl. Phys. Lett. 103, 242414 (2013).

[32] H. L. Wang, C. H. Du, Y. Pu, R. Adur, P. C. Hammel, and F. Y. Yang, Phys. Rev. Lett. 112, 197201 (2014).

[33] C. F. Pai, Y. Ou, L. H. Vilela-Leão, D. C. Ralph, and R. A. Buhrman, Phys. Rev. B 92, 064426 (2015).

[34] W. Zhang, W. Han, X. Jiang, S.-H. Yang, and S. S. P. Parkin, Nat. Phys. 11, 496 (2015).

[35] V. Vlaminck, J. E. Pearson, S. D. Bader, and A. Hoffmann, Phys. Rev. B 88, 064414 (2013).

[36] K. Ando, Y. Kajiwara, K. Sasage, K. Uchida, and E. Saitoh, IEEE Trans. Magn. 46, 1331 (2010).

[37] S. Isogami, M. Tsunoda, M. Oogane, A. Sakuma, and M. Takahasi, Appl. Phys. Ex. 6, 063004 (2013).

[38] See Supplemental Material at http://link.aps.org/supplemental/ 10.1103/PhysRevB.93.064408 for additional measurement data.

[39] See http://micromagnum.informatik.uni-hamburg.de for MicroMagnum.

[40] For simulations the following parameters were used: Saturation magnetization $M_{s}=800 \mathrm{kA} \mathrm{m}^{-1}$, damping constant $\alpha=0.01$, exchange constant $A=1.3 \times 10^{-11} \mathrm{~J} \mathrm{~m}^{-1}$, and anisotropy constant $K=0 \mathrm{~J} \mathrm{~m}^{-3}$. The cell sizes are $4 \times 4 \times 30 \mathrm{~nm}^{3}$.

[41] J. P. Park, P. Eames, D. M. Engebretson, J. Berezovsky, and P. A. Crowell, Phys. Rev. Lett. 89, 277201 (2002).

[42] K. Y. Guslienko, R. W. Chantrell, and A. N. Slavin, Phys. Rev. B 68, 024422 (2003).

[43] Y. S. Gui, L. H. Bai, and C.-M. Hu, Sci. Chin. Phys. Mech. Astron. 56, 124 (2013).

[44] H. Chen, X. Fan, H. Zhou, W. Wang, Y. S. Gui, C.-M. Hu, and D. Xue, J. Appl. Phys. 113, 17C732 (2013).

[45] L. Bai, P. Hyde, Y. S. Gui, C.-M. Hu, V. Vlaminck, J. E. Pearson, S. D. Bader, and A. Hoffmann, Phys. Rev. Lett. 111, 217602 (2013).

[46] V. E. Demidov, S. Urazhdin, H. Ulrichs, V. Tiberkevich, A. Slavin, D. Baither, G. Schmitz, and S. O. Demokritov, Nat. Mater. 11, 1028 (2012).

[47] L. Liu, T. Moriyama, D. C. Ralph, and R. A. Buhrman, Phys. Rev. Lett. 106, 036601 (2011).

[48] K. Ando, S. Takahashi, K. Harii, K. Sasage, J. Ieda, S. Maekawa, and E. Saitoh, Phys. Rev. Lett. 101, 036601 (2008).

[49] Y. S. Gui, A. Wirthmann, and C.-M. Hu, Phys. Rev. B 80, 184422 (2009).

[50] P. Anderson and H. Suhl, Phys. Rev. 100, 1788 (1955). 